\title{
Liouville equation under perturbation
}

\author{
L.A. Kalyakin \\ Institute of Mathematics, Ufa Sci. Centre, of Russian Acad. of Sci. \\ Institute of Mathematics, 112,Chernyshevsky str., Ufa, \\ 450000, Russia \\ E-mail: klen@imat.rb.ru *
}

December, 16, 1999

\begin{abstract}
Small perturbation of the Liouville equation under smooth initial data is considered. Asymptotic solution which is available for a long time interval is constructed by the two scale method.
\end{abstract}

The Cauchy problem for the Liouville equation with a small perturbation

$$
\begin{gathered}
\partial_{t}^{2} u-\partial_{x}^{2} u+8 \exp u=\varepsilon \mathbf{F}[u], \quad 0<\varepsilon \ll 1, \\
\left.u\right|_{t=0}=\psi_{0}(x),\left.\partial_{t} u\right|_{t=0}=\psi_{1}(x), \quad x \in \mathbb{R}
\end{gathered}
$$

is considered. The problem is not suit under soliton perturbation theory because the Liouville equation has no any soliton solution. Initial functions are here arbitrary, ones are smooth and decay rapidly at infinity $\psi_{0}, \psi_{1}(x)=\mathcal{O}\left(x^{-N}\right),|x| \rightarrow$ $\infty, \forall N$. So we deal with a smooth solution; the case of singular solutions was considered in [1].

The perturbation operator is determined by two smooth functions $F_{1}, F_{2}$ :

$$
\mathbf{F}[u]=\partial_{x} F_{1}\left(\partial_{x} u, \partial_{t} u\right)+\partial_{t} F_{2}\left(\partial_{x} u, \partial_{t} u\right) .
$$

The purpose is to construct an asymptotic approach of the solution $u(x, t ; \varepsilon)$ as $\varepsilon \rightarrow 0$ uniformly over long time interval $\left\{x \in \mathbb{R}, 0<t \leq \mathcal{O}\left(\varepsilon^{-1}\right)\right\}$.

Results. 1. The solution of the unperturbed problem (as $\varepsilon=0$ ) decomposes asymptotically at infinity (as $t \rightarrow \infty$ ) on two simple waves which travel on a decreasing background $u(x, t ; 0)=-4 t+A_{ \pm}^{0}\left(s^{ \pm}\right)+\mathcal{O}\left(\left(s^{\mp}\right)^{-N}\right), s^{\mp} \rightarrow \mp \infty$. 2. The structure of the asymptotic solution as $\varepsilon \rightarrow 0$ remains the same $u(x, t ; \varepsilon) \approx$ $-4 t+A_{ \pm}\left(s^{ \pm}, \tau\right)+\mathcal{O}(\varepsilon)$ for long times $t \approx \varepsilon^{-1}$. The perturbation affects only a slow deformation of the waves $A_{ \pm}=A_{ \pm}\left(s^{ \pm}, \tau\right)$ on the slow time scale $\tau=\varepsilon t$. 3. The deformation of the waves is described by the first order PDE's

$$
\pm 2 \partial_{\tau} A_{ \pm}=H_{ \pm}\left(\partial_{s} A_{ \pm}\right), \quad s \in \mathbb{R}, \tau>0
$$

*This research has been supported by the Russian Foundation of the Fundamental Research under Grants 99-01-00139, 96-15-96241 
where $H_{ \pm}(B)=F_{1}(B,-4 \pm B)-F_{1}(0,-4) \pm F_{2}(B,-4 \pm B) \mp F_{2}(0,-4)$. The initial data are here taken from the fast time asymptotics of the unperturbed solution

$$
A_{ \pm}(s, 0)=A_{ \pm}^{0}(s)
$$

This result is very close to the case of linear wave under perturbation, which slow deformation is described by the Hopf equation, [2].

We use here the two scale method and construct an asymptotic solution as a piece of the asymptotic series

$$
u \approx \sum_{n=0}^{\infty} \varepsilon^{n} \stackrel{n}{u}(x, t, \tau), \tau=\varepsilon t, \quad \varepsilon \rightarrow 0 .
$$

Remark. The direct asymptotic expansion $u \approx \sum \varepsilon^{n} \stackrel{n}{u}(x, t)$ does not provide approach to the solution over long time interval $t \approx \mathcal{O}\left(\varepsilon^{-1}\right)$ through the secular terms in corrections.

\section{Unperturbed problem $(\varepsilon=0)$}

General solution of the nonlinear equation

$$
\partial_{t}^{2} \phi-\partial_{x}^{2} \phi+8 \exp \phi=0,(x, t) \in R^{2}
$$

is given (Liouville, [3]) by the formula

$$
\phi(x, t)=\ln \frac{r_{+}^{\prime}\left(s^{+}\right) r_{-}^{\prime}\left(s^{-}\right)}{r^{2}\left(s^{+}, s^{-}\right)}, \quad r=r_{+}\left(s^{+}\right)+r_{-}\left(s^{-}\right), s^{ \pm}=x \pm t
$$

with arbitrary functions $r_{ \pm}$. Initial conditions (0.2) give two ODE's for the $r_{ \pm}$ which may be linearized by change of variable $r_{ \pm}^{\prime}=w / \rho_{ \pm}^{2},(\forall w=$ const $\neq 0)$, so that

$$
\rho_{ \pm}^{\prime \prime}-\Psi_{ \pm}(x) \rho_{ \pm}=0 .
$$

The potentials $\Psi_{ \pm}(x)$ are determined by the original initial data, [4]

$$
\Psi_{ \pm}(x)=\exp \left(\psi_{0}\right)+\left(\frac{\psi_{0}^{\prime} \pm \psi_{1}}{4}\right)^{2}-\frac{1}{4}\left(\psi_{0}^{\prime} \pm \psi_{1}\right)^{\prime} .
$$

So the Cauchy problem for the Liouville equation is integrable.

Lemma 1.1 Let as $\psi_{0}, \psi_{1}(x)=\mathcal{O}\left(x^{-N}\right),|x| \rightarrow \infty, \forall N$, and equations (1.2) are not on spectrum. Than the solution of the Cauchy problem for the Liouville equation has an asymptotics

$$
\phi(x, t)=-4 t+A_{ \pm}\left(s^{ \pm}\right)+\mathcal{O}\left(\left(s^{\mp}\right)^{-N}\right)+\mathcal{O}\left(e^{-4 t}\right), s^{\mp} \rightarrow \mp \infty, t \rightarrow \infty
$$

with the matching property

$$
A_{ \pm}(s)=\left\{\begin{array}{l}
-\ln a^{2}+\mathcal{O}\left(s^{-N}\right), s \rightarrow \pm \infty, \quad(a=\text { const } \neq 0) \\
\mathcal{O}\left(s^{-N}\right), s \rightarrow \mp \infty
\end{array}\right.
$$


Functions $A_{ \pm}(s)$ are reading from $r_{ \pm}(s)$. If the $r_{ \pm}$are fixed by the conditions at infinity

$$
\begin{gathered}
r_{ \pm}(x)=(1 / a) \exp (2 x)\left[1+\mathcal{O}\left(x^{-N}\right)\right], \quad x \rightarrow-\infty \\
r_{ \pm}(x)=a \exp (2 x)\left[1+\mathcal{O}\left(x^{-N}\right)\right], \quad x \rightarrow+\infty
\end{gathered}
$$

then

$A_{+}\left(s^{+}\right)=\ln \left[\exp \left(2 s^{+}\right) r_{+}^{\prime}\left(s^{+}\right) / a r_{+}^{2}\left(s^{+}\right)\right], \quad A_{-}\left(s^{-}\right)=\ln \left[\exp \left(-2 s^{-}\right) r_{-}^{\prime}\left(s^{-}\right) / a\right]$.

Functions $A_{ \pm}(s)$ do not depend on the choice of $r_{ \pm}(s)$ within the Bianchi transform [5] and may be used for parametrization of the general solution.

Corollare 1.1 General solution of the Liouville equation can be parametrized by the pare of functions $A_{ \pm}(s)$ which have the matching property (1.3) so that

$$
\phi(x, t)=\Phi\left[A_{+}, A_{-}\right] \equiv \ln \frac{r_{+}^{\prime} r_{-}^{\prime}}{\left(r_{+}+r_{-}\right)^{2}}
$$

in view of (1.6).

\section{Linearized problem for the correction}

Corrections $\stackrel{n}{u}(n \geq 1)$ are obtained from linear equations with corresponding initial conditions

$$
\partial_{t}^{2} \stackrel{n}{u}-\partial_{x}^{2} \stackrel{n}{u}+8 \frac{r_{+}^{\prime} r_{-}^{\prime}}{r^{2}} \stackrel{n}{u}=\stackrel{n}{f}(x, t ; \varepsilon),\left.\quad \stackrel{n}{u}\right|_{t=0}=\stackrel{n}{\psi_{0}}(x),\left.\partial_{t} \stackrel{n}{u}\right|_{t=0}=\stackrel{n}{\psi_{1}}(x) .
$$

The right sides are here determined by the previous approaches. Dependence on the fast variables $x, t$ is only determined from these equations.

General solution of the homogeneous linear equation is given by the formula

$$
u_{0}(x, t ; \varepsilon)=\frac{j_{+}^{\prime}}{r_{+}^{\prime}}+\frac{j_{-}^{\prime}}{r_{-}^{\prime}}-2 \frac{j_{+}+j_{-}}{r}
$$

where $j_{ \pm}=\stackrel{n}{=} j_{ \pm}\left(s^{ \pm}\right)$are arbitrary functions. In context of the Cauchy problem they are determined by the initial data. In this way pare linear ODE's are obtained which can be solved in explicit form.

A similar formula with $j_{ \pm}\left(s^{ \pm}, t\right)$ may be used to solve the nonhomogeneous linear equation (2.1). The functions $j_{ \pm}(x, t)$ are defined from ODE's as well, so that the solution is represented by the integral

$$
u(x, t)=\int_{s^{-}}^{s^{+}} \int_{s^{-}}^{\sigma^{+}} K\left(s^{+}, s^{-}, \sigma^{+}, \sigma^{-}\right) f\left(\sigma^{+}, \sigma^{-}\right) d \sigma^{-} d \sigma^{+}
$$


taken over the characteristic triangle. The kernel $K$ is here expressed by means of $r_{ \pm}$

$$
\begin{gathered}
K\left(s^{+}, s^{-}, \sigma^{+}, \sigma^{-}\right)=\frac{1}{2 r\left(s_{+}, s_{-}\right) r\left(\sigma_{+}, \sigma_{-}\right)} \\
\left\{r_{+}\left(s^{+}\right) r_{-}\left(s^{-}\right)+r_{+}\left(\sigma^{+}\right) r_{-}\left(\sigma^{-}\right)+\frac{1}{2}\left[r_{+}\left(s^{+}\right)-r_{-}\left(s^{-}\right)\right]\left[r_{+}\left(\sigma^{+}\right)-r_{-}\left(\sigma^{-}\right)\right]\right\} .
\end{gathered}
$$

Lemma 2.1 Let both the right side and the initial functions decay rapidly at infinity. Than the solution of the Cauchy problem for linearized equation is bounded and has an asymptotics

$$
\begin{gathered}
u(x, t)=U_{ \pm}\left(s^{ \pm}\right)+\mathcal{O}\left(\left(s^{\mp}\right)^{-N}\right), s^{\mp} \rightarrow \mp \infty, \\
U_{ \pm}(s)=\left\{\begin{array}{l}
U+\mathcal{O}\left(s^{-N}\right), s \rightarrow \pm \infty, \quad(U=\text { const }), \\
\mathcal{O}\left(s^{-N}\right), s \rightarrow \mp \infty .
\end{array}\right.
\end{gathered}
$$

\section{$3 \quad$ Perturbed problem $(\varepsilon \neq 0)$}

We construct a formal asymptotic solution in the form (0.6) where the leading order term is taken as a solution of the unperturbed equation. The original idea is to use $A_{ \pm}$- parametrization of this solution ${ }_{u}^{0}=\Phi\left[A_{+}, A_{-}\right]$as it was pointed in (1.7). The second idea becomes from the two scale method. It is assumed the functions $A_{ \pm}\left(s^{ \pm}, \tau\right)$ depend on both fast $s^{ \pm}=x \pm t$ and slow $\tau=\varepsilon t$ variables. The initial values for the $A_{ \pm}\left(s^{ \pm}, \tau\right)$ as $\tau=0$ are taken in (0.5) from the unperturbed solution. Ones are calculated per the initial function $\psi_{0}, \psi_{1}(x)$ from the equations $(1.2),(1.4),(1.6)$.

Dependence on the slow variable as $\tau>0$ is determined by the differential equations obtained from the secular condition which means the first order correction is small: $\varepsilon \stackrel{1}{u}=\mathrm{o}(1), \varepsilon \rightarrow 0, \tau=\varepsilon t$ uniformly over long time interval $0<t \leq \mathcal{O}\left(\varepsilon^{-1}\right)$.

The right side of the first order equation is given by formula

$$
\stackrel{1}{f}(x, t, \tau)=\mathbf{F}[\stackrel{0}{u}]-2 \partial_{\tau} \partial_{t} \stackrel{0}{u} .
$$

One can see from the lemma 2.1 that the secular condition can be formulated through the right side as follows: The right side tends to zero at infinity as $s^{ \pm} \rightarrow 0$. This requirement gives two equations

$$
\pm 2 \partial_{\tau} \partial_{s} A_{ \pm}=\mathbf{F}\left[-4 t+A_{ \pm}\right] .
$$

If we integrate these relations taking into account boundary conditions

$$
A_{ \pm}\left(s^{ \pm}, \tau\right) \rightarrow 0 \quad \text { as } \quad s^{ \pm} \rightarrow \mp \infty
$$

than the first order PDE's (0.4) are obtained. 
Lemma 3.1 1. If the perturbation operator has the form (0.3) than the Cauchy problem for deformation equations (0.4),(0.5) has the unique smooth solution on some finite interval $0 \leq \tau \leq \tau_{0}$. 2. Under such functions $A_{ \pm}\left(s^{ \pm}, \tau\right)$ the secular condition is hold for the first correction, i.e. $\stackrel{1}{u}(x, t, \tau)$ is bounded uniformly for all $x, t \in \mathbb{R}^{2}, \tau \in\left[0, \tau_{0}\right]$.

Corollare 3.1 The function $U_{1}(x, t, \varepsilon)=\stackrel{0}{u}(x, t, \varepsilon t)+\varepsilon \stackrel{1}{u}(x, t, \varepsilon t)$ under substitution in the equations (0.1),(0.2) gives a remainder of order $\mathcal{O}\left(\varepsilon^{2}\right)$ uniformly for all $x \in \mathbb{R}, 0 \leq t \leq \tau_{0} \varepsilon^{-1}$.

Theorem 3.1 Let the perturbation operator has the form (0.3) and the initial functions in (0.2) are such that equations (1.2) are not on spectrum (i.e. in general position). Than the leading order term of the formal asymptotic solution for the perturbed problem (0.1),(0.2) is given by the Liouville formula (1.1) in which the functions $r_{ \pm}\left(s^{ \pm}, \tau\right)$ depend on the additional slow time $\tau=\varepsilon t$. Slow deformation of the $r_{ \pm}\left(s^{ \pm}, \tau\right)$ is determined from equations (0.4),(0.5),(1.6),(1.4).

\section{References}

[1] L.A. Kalyakin, Teoret. Matemat. Fisika 118, 3 (1999) 390-396 (in Russian).

[2] L.A. Kalyakin, Math. USSR Sbornik 52, 1 (1985) 91-114 Matem. Sbornik. 124, 1 (1984) 96-120 (in Russian).

[3] J. Liouville, Journ. math. pure et appl. 18 (1853) 71-74.

[4] G.P.Jeorjadze, A.K. Pogrebkov, M.C. Polivanov, Teoret. Matemat. Fisika 40, 2 (1979) 221-234 (in Russian).

[5] L. Bianchi, Ann. Sci. Norm. Super. Piza, Ser 1, 2 (1879) 26. 